\title{
La evolución de la empleabilidad de la mujer en el mercado de trabajo español ${ }^{1}$
}

\author{
Marina Hernando González ${ }^{2}$ \\ Universidad Complutense de Madrid \\ Pilar Zarzosa Espina ${ }^{3}$ \\ Universidad de Valladolid
}

\section{Artículo científico}

Material original autorizado para su primera publicación en el Journal de Ciencias Sociales, Revista Académica de la Facultad de Ciencias Sociales de la Universidad de Palermo.

\section{Recepción: 20-09-2017}

\section{Aceptación: 07-02-2018}

Resumen: En el presente trabajo se estudia la evolución de la empleabilidad femenina en el mercado de trabajo español y los distintos determinantes que afectan a las mujeres a la hora de decidir si seguir trabajando o no. Así pues, en primer lugar, se realiza un estudio descriptivo sobre el tema y se revisan distintos artículos con objetivos similares, para concluir que la situación de las mujeres en el mercado de trabajo ha mejorado. Sin embargo, el salario femenino, en términos generales, continúa siendo inferior al de los hombres y, además, muchas mujeres ocupan puestos de trabajo a tiempo parcial ya que, en su mayoría, son ellas las que se ocupan de las tareas no remuneradas. En segundo lugar, se propone y se estima, mediante mínimos cuadrados ordinarios (MCO), el modelo econométrico que mejor explica la relación a largo plazo entre las variables explicativas —número medio de hijos, mujeres que trabajan a tiempo parcial y mujeres que han alcanzado estudios superiores y la variable dependiente, el número de mujeres que tienen un empleo, entre los años 1987 y 2015 . Para ello, se estudian los problemas de no estacionariedad de las variables y se propone una relación de cointegración. Por último, se obtiene un modelo no espurio, con un buen ajuste y sin autocorrelación, donde el número medio de hijos por mujer influye positivamente sobre la variable dependiente y las otras dos variables explicativas se comportan de forma diferente según se vean o no afectadas por la variable ficticia introducida por un cambio estructural.

\footnotetext{
${ }_{1}^{1}$ Agradecemos a los revisores/as anónimos de la revista sus valiosas sugerencias, que hemos llevado a cabo y, sin lugar a dudas, han servido para mejorar el contenido de este trabajo.

${ }^{2}$ Estudiante del Máster en Estadísticas Oficiales e Indicadores Sociales y Económicos. Correo electrónico: marhern13@gmail.com

${ }^{3}$ Departamento de Economía Aplicada.Sección de Estadística y Econometría. Correo electrónico: pzarzosa@eaee.uva.es
} 
Palabras clave: empleabilidad femenina; mercado de trabajo español, modelo econométrico; determinantes.

\section{The evolution of the employability of women in the Spanish labor market}

Abstract:This paper focuses on the evolution of female employability in Spanish labour market, as well as, the different determinants that affect women upon deciding to keep working or not. In order to do this, a descriptive study about the subject is carried out and articles with similar objectives are revised, concluding that the situation of women in labour market has improved. However, women's wages, in general terms, are still lower than men's and, furthermore a lot of women occupy part-time jobs, due to the fact that they are the ones who are in charge of unpaid tasks. Secondly, the econometric model that best explains the longterm relationships between the studied variables is put forward and estimated, by means on ordinary least squares (OSL): number of children, women who have part-time jobs and who have achieved higher studies and the dependent variable, number of employed women, between the years 1987 and 2015. For this, the problems of non-stationarity of the variables are studied and a cointegration relation is proposed. Last of all, it gets a non-spurious model, with a good fit and no autocorrelation, finding that the average number of children per woman affects positively the dependent variable and the other two explicative variables behave on a different way as they are affected by the dummy variable introduced by structural change.

Keywords: female employability; spanish labour market, econometric model; determinants.

\section{Introducción}

La situación de las mujeres en el mercado laboral español ha ido evolucionando de forma paulatina y desigual a lo largo de los años (Cebrián y Moreno, 2008). Actualmente, según los datos de Eurostat, la tasa de ocupación de las mujeres para el año 2016 es de 54,3 \% frente al 32,8 \% del año 1996.

Este aumento ha generado un importante impacto social y cultural, lo que modificó el rol de la mujer dentro de la unidad familiar y la distribución de las tareas del hogar, que ahora es más equitativa (Millán, Santos y Pérez, 2015).

Para una mejor comprensión de la situación y de las causas de la desigualdad laboral entre hombres y mujeres, se inicia el trabajo con un estudio de la literatura existente sobre el tema. Seguidamente se analiza la evolución de la empleabilidad de las mujeres en el mercado de trabajo desde el año 1976 hasta la actualidad y se hace hincapié en los motivos que hacen que las mujeres continúen en el mercado laboral. A continuación, se compara la situación laboral femenina con la de otros países europeos y, además, se estudian las principales 
causas de la diferencia salarial entre hombres y mujeres y de la gran participación de las mujeres en empleos a tiempo parcial.

El principal objetivo del trabajo es estudiar, a través de un modelo econométrico, la evolución y la influencia de determinados factores que afectan a las mujeres que están trabajando. Para identificar dichos factores se parte de las investigaciones que perseguían objetivos similares, y que han sido estudiadas en la primera parte de este trabajo. Seguidamente, para especificar el modelo, se han seleccionado diversos factores de entre varios posibles, como la población activa femenina, el nivel de educación alcanzado, la población ocupada femenina o las mujeres que trabajan a tiempo parcial. Algunos, como el estado civil o la tasa de empleo femenina según tenga o no hijos, no se pudieron introducir por falta de datos. Finalmente, las variables explicativas elegidas fueron el número medio de hijos por mujer, el porcentaje de mujeres que han alcanzado estudios superiores y el número de mujeres que trabajan a tiempo parcial.

Este estudio se realiza con datos anuales desde 1987 hasta 2015. Finalmente, se propone y se estima el modelo que mejor explique la relación entre dichas variables, para lo que se utilizan distintas técnicas econométricas.

\section{Perspectiva teórica}

A pesar de los avances conseguidos, muchas mujeres hoy siguen ocupando puestos de trabajo peor remunerados y cualificados que los hombres, con una mayor presencia en trabajos temporales. Además, la brecha de género aumenta con respecto a las tasas de empleo y de paro. Esto trae como consecuencia una menor independencia económica actual y futura de la mujer.

Por otro lado, según el Instituto Nacional de Estadística (INE), existen distintos motivos por los que los hombres y las mujeres deciden no incorporarse al mercado de trabajo. Mientras que los hombres lo hacen principalmente por motivos de estudio y enfermedad/incapacidad, las mujeres lo hacen fundamentalmente por el hogar. Lo que demuestra que todavía las mujeres tienen mayores dificultades que los hombres a la hora de conciliar la vida laboral con la familiar (Cebrián y Moreno, 2013). En concreto, según los datos del INE, el número de mujeres inactivas por labores del hogar en España, para el año 2016, era de 3406,7 frente a los 377,9 hombres inactivos en el mismo momento. 


\subsection{Antecedentes bibliográficos}

Existen muchos trabajos que ya han estudiado la relación que puede existir entre la participación de la mujer en el mercado de trabajo y diferentes factores como el nivel de estudios o el número de hijos en el ámbito español e internacional.

La mayoría de estos trabajos se centran en el estudio de las razones que hacen o no participar a la mujer en el mercado de trabajo para un grupo de mujeres en un año concreto. Utilizan un modelo probabilístico tipo logit binomial, donde la variable dependiente es binaria. Esta variable toma dos valores: 1 si la mujer participa en el mercado laboral y 0 si no participa. Es el caso de los trabajos de Guevara (2014), que se centra en la región de Santiago de Cali, De Pablos (sin fecha) o Martínez (2001) que se focaliza en España. En ambos se concluye que las variables que tienen una mayor influencia, a la hora de tomar la decisión de participar son: el nivel educativo, la riqueza del hogar, cuidado de niños, estar casada o viuda y en el caso del primer trabajo, los valores culturales, como el índice de machismo.

También se han desarrollado este tipo de estudios para comprobar si el efecto que tienen distintas variables es o no el mismo en distintas regiones. Es el caso del artículo de Ferrada y Zarzosa (2010), en el que se estudia cómo distintas variables pueden tener un impacto diferente en cada región de Chile, excepto el nivel educativo, que influye de forma positiva en la participación laboral femenina en todas las regiones del país.

Otro ejemplo es el trabajo de González, Prieto y Pérez (1999), que compara la participación laboral femenina entre la comunidad de Castilla y León y España, donde, nuevamente, mujeres con mayor formación tienen una probabilidad más alta de participar en el mercado de trabajo y las mujeres casadas y con hijos tienen una probabilidad menor.

En el trabajo de Alonso, Fernández y Rincón (2015), las autoras no se limitan a estudiar los efectos que influyen en la decisión sobre participar en el mercado laboral español, sino que también analizan los factores que influyen en la decisión de una mujer de tener un hijo o no. Los autores estudian ambas variables mediante un modelo probit bivariante, ya que se ha tenido en cuenta que puede existir relación entre las variables inobservables de cada modelo.

\subsection{Evolución de la participación y empleabilidad de las mujeres en el mercado} laboral español

La incorporación de la mujer en el mercado de trabajo ha estado marcada por diferentes factores económicos, sociales y políticos. A partir del año 1984 se produjo un ingreso masivo de la mujer al mercado laboral español. Esto fue posible gracias a una serie de medidas que lo fomentaron, como la promulgación de la Constitución Española en 1978, 
que fue el inicio para superar las desigualdades por razón de sexo. Asimismo, en 1983 fue ratificada en España la Convención sobre la Eliminación de Todas las Formas de Discriminación contra la Mujer (Millán, Santos y Pérez, 2015).

En 1986, se produce de nuevo un gran aumento del empleo, que fue posible gracias a la entrada de España en la Unión Europea. Entre los años 1991 y 1994, España sufre las consecuencias de una crisis económica mundial, acentuada por problemas internos del país. En esta etapa, se produce una gran destrucción de empleo, la cual fue más intensa entre los varones. Sin embargo, se observa una tendencia creciente en la población activa femenina (Cebrián y Moreno, 2008).

Desde 1994 hasta 2007 se da una etapa de gran crecimiento económico, donde el empleo de las mujeres se multiplicó por dos. Un avance importante es la promulgación de la Ley orgánica 3 de 2007, que de acuerdo con Millán, Santos y Pérez (2015) favorece la igualdad efectiva entre hombres y mujeres, reconociendo el derecho a la conciliación de la vida personal, familiar y laboral. Además, el sector de servicios crece, de forma que las mujeres tienen nuevas oportunidades de empleo.

Sin embargo, a partir del año 2008 se produce una destrucción masiva del empleo, tanto masculino como femenino, provocada por la gran crisis económico-financiera. Como consecuencia, el número de parados en 2013 llegó a sobrepasar los seis millones.

Esto ha dado lugar a empleos con salarios más bajos y de una gran rotación, lo que perjudica más a las mujeres que a los hombres, ya que el colectivo femenino es más sensible a las fluctuaciones de la economía (Millán, Santos y Pérez, 2015).

Para mejorar esta situación, se lleva a cabo la reforma laboral de 2012. Fue un primer avance para dotar al mercado de una mayor flexibilidad e "intentar disminuir los desequilibrios del mercado laboral español a través del fomento de la empleabilidad de los trabajadores, la adaptación de los salarios, así como de las condiciones laborales" (Elneser, 2015, p. 12).

A partir del año 2014, parece que la economía española comenzó a recuperarse, ya que aumentó el número de mujeres como de hombres ocupados y disminuyeron las tasas de paro para ambos sexos.

Esta evolución se plasma en el gráfico siguiente, que recoge el número de mujeres activas y ocupadas en España a lo largo del periodo analizado. 
Gráfico 1: Evolución de la población activa y ocupada femenina desde 1995 hasta 2015.

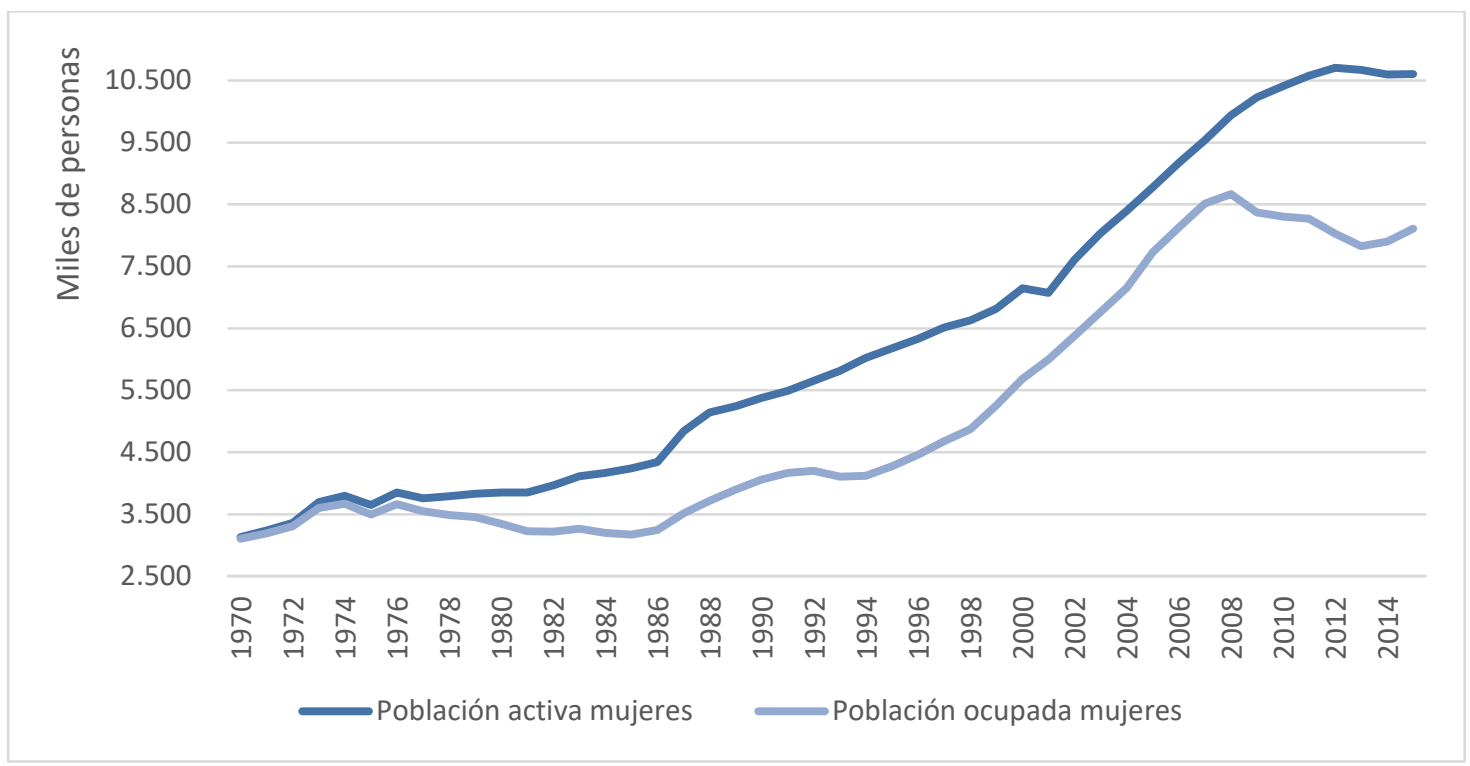

Fuente: Elaboración propia a través de la OECD.

\subsection{España frente a otros países}

Pese al aumento de las tasas ocupación de las mujeres españolas en los últimos años, estas se encuentran a la cola de la Unión Europea respecto a la tasa de empleo femenino para la población comprendida entre los 15 y 64 años de edad. En concreto, se sitúan en el puesto 23 sobre el total de los 28 países miembros, con una tasa de empleo del $54,3 \%$ para el año 2016. En este tema, España está por encima solamente de Grecia (43,3 $\%)$, Italia (48,1\%), Croacia (52,4\%), Malta (52,6\%) y Rumanía (53,3\%); se sitúa muy por detrás de los países del norte de Europa, como Suecia (74,8 \%) o Noruega (72,8 \%).

La brecha salarial de género es un problema que permanece tanto en Europa como en España (Hernández, 2015), aunque es más acusado en dicho país. Actualmente, como afirma García (2016), se encuentra por debajo de la media europea y por debajo de países como Alemania, aunque por encima de Italia.

En cuanto al trabajo a tiempo parcial, ha crecido en la última década en todos los países de la Unión Europea, aunque tuvo una mayor incidencia en el caso de España, ya que aumentó en mayor medida. Tanto en la Unión Europea como en España, afecta más a las mujeres casadas y con hijos. Sin embargo, la causa principal de la elección de esta jornada para las mujeres europeas es el cuidado de niños o adultos enfermos, incapacitados o mayores, y no por no poder encontrar trabajo de jornada completa, como ocurre en el caso 
español. Este rasgo es común a otros países del sur de Europa, como Italia, pero no se da en países del norte de Europa, donde el apoyo institucional a la incorporación de la mujer al trabajo es mucho mayor. (Ortiz, 2014).

\subsection{Causas del aumento de la empleabilidad de las mujeres en España}

A pesar de todo lo ocurrido, desde el año 1976 la población ocupada femenina ha crecido. Según Cebrián y Moreno (2008), dicho aumento se debe a diferentes factores:

- Mayor inversión en capital humano. Las mujeres con menos de 20 años de edad han disminuido su presencia en el mercado laboral, puesto que se prolongan los años de estudio, tanto obligatorios como superiores. Sin embargo, una mayor cualificación de las mujeres hace aumentar su empleabilidad en el mercado laboral, puesto que aumenta el coste de oportunidad de permanecer en casa, ya que los salarios de las mujeres cualificadas son mayores. Esto supone que la tasa de empleabilidad se parece más a la de los hombres, lo que da lugar a una mayor permanencia de la mujer en el mercado laboral.

- Disminución de la natalidad y aumento en la edad media de maternidad. Muchas mujeres deciden reducir el número de hijos para poder trabajar con una frecuencia mayor y alcanzar sus metas profesionales, lo que lleva consigo el retraso de la edad a la que la mujer tiene el primer hijo.

Por otro lado, el tiempo de trabajo y su distribución son factores determinantes a la hora de analizar el empleo femenino. Tal y como indica el informe "La situación de las mujeres en el mercado de trabajo en 2016" elaborado por la Secretaría de Estado de Empleo, las mujeres ocupan las tres cuartas partes del empleo a tiempo parcial.

Se puede definir el trabajo a tiempo parcial, según el artículo 12 del Estatuto de los Trabajadores, como "aquel en el que se haya acordado la prestación de servicios durante un número de horas al día, a la semana, al mes o al año, inferior a la jornada de trabajo de un trabajador a tiempo completo comparable" (Real Decreto Legislativo, 2/2015). Este concepto, como manifiesta Ortiz (2014), se puso en marcha como parte de la estrategia flexibilizadora para promover la incorporación al empleo a las mujeres y para adecuar la producción a las necesidades del mercado. 
Sin embargo, y según los datos obtenidos de Eurostat para el año 2016, el motivo principal por el cual las mujeres, al igual que los hombres, trabajan a tiempo parcial es por no encontrar un trabajo de jornada completa. La segunda razón por la que las mujeres eligen esta jornada es para compaginar el trabajo con el cuidado y atención a personas dependientes. Sin embargo, no ocurre lo mismo en el caso de los hombres ya que, la segunda razón por la que trabajan en este tipo de jornada es por seguir en cursos de enseñanza o formación; las tareas de cuidado de otras personas son un motivo menor.

Esto ha dado lugar a la inestabilidad del colectivo femenino, originando unas condiciones, tanto presentes como futuras, discriminatorias en muchos aspectos, como por ejemplo en la pensión de jubilación contributiva. Otro factor que influye sobre las mujeres que deciden trabajar es el salario. Tal y como afirma el INE, la brecha salarial de género (no ajustada a las características individuales) se puede definir como "la diferencia entre el salario bruto por hora de los hombres y el de las mujeres, expresado como porcentaje del salario bruto por hora de los hombres" (INE, 2017).

Las mujeres tienen por media unos ingresos salariales anuales menores a los de los varones. Además, la brecha entre hombres y mujeres crece conforme aumenta la edad; los ingresos de los hombres siempre son mayores a los de las mujeres (Cebrián y Moreno, 2008).

En la actualidad los problemas de la empleabilidad laboral de las mujeres ocasionados por la diferencia salarial entre los sexos se deben a diferentes causas. Las más relevantes, según indican la Comisión Europea en su informe "Cómo combatir la brecha salarial entre hombres y mujeres en la Unión Europea” (2014) y la Organización Internacional del Trabajo (OIT) en su informe "Igualdad salarial" (2013) son:

- Segregación laboral. Tradicionalmente, las mujeres se han concentrado en sectores peor remunerados y menos variados que los hombres. Esto se conoce como segregación profesional horizontal. Como afirman Salinas y Romaní (2016), los estereotipos van unidos a los factores culturales y el comportamiento de los hombres y las mujeres varía según éstos.

- Búsqueda de trabajos que les permitan conciliar su vida laboral con la familiar, ya que siguen siendo ellas las que tienen una mayor carga en las tareas no remuneradas. Por ello existe un gran número de mujeres que realizan trabajos a tiempo parcial con un salario más bajo o que directamente deciden renunciar a su vida profesional. 
- Discriminación salarial. En la práctica existen sistemas que por razón de sexo pueden ofrecer distintas formas de remuneración, más allá del salario base, como por ejemplo las horas extraordinarias o la antigüedad. Asimismo, las mujeres pueden tener más interrupciones a lo largo de su carrera profesional, lo que en ciertas ocasiones las hace abandonar el mercado laboral. Estos hechos contribuyen a la discriminación indirecta que hace que las mujeres se vean más limitadas para alcanzar puestos de trabajo de mayor retribución.

Todo esto hace que las mujeres se incorporen al mercado laboral en condiciones de desventaja, ya que su coste de oportunidad de tener un trabajo es mayor al de permanecer en casa y por ello muchas mujeres optan por esto último.

\section{Metodología y resultados}

\subsection{Variables.}

El principal aporte de este trabajo es estudiar mediante un modelo de regresión general, la empleabilidad femenina en el mercado de trabajo español entre los años 1987 y 2015. Por ello, se han seleccionado series anuales de datos temporales de dos bases de datos distintas: la Encuesta Europea de Fuerza del Trabajo (LFS) de Eurostat y el Banco Mundial. Posteriormente, se realizarán distintos análisis para llegar al modelo que mejor explique el comportamiento de dicha magnitud a lo largo del tiempo.

Son muchas las variables que afectan a las mujeres que están trabajando. En este trabajo se estudiarán aquellas que son más relevantes según la literatura analizada.

La variable dependiente elegida es la población ocupada femenina (ocupadas) en España, es decir las mujeres de 16 años o más que tienen un empleo o que ejercen una actividad por cuenta propia. En cuanto a las variables explicativas del modelo, se han incluido fundamentalmente aquéllas que son significativas para explicar la relación con la variable dependiente. Éstas son:

mparcial. Mujeres que trabajan a tiempo parcial. Definida como el número de mujeres de 15 a 64 años que trabajan a tiempo parcial. Expresada en miles de mujeres.

educación. Tasa bruta de matrícula femenina en nivel superior. Refleja el número total de mujeres matriculadas en educación superior con respecto a la población total de mujeres en el grupo etario cinco años después de finalizar la enseñanza secundaria.

ICF. Indicador Coyuntural de Fecundidad. "Mide el número medio de hijos que tendría una mujer perteneciente a un determinado ámbito a lo largo de su vida fértil en caso de mantener la misma intensidad fecunda por edad que la observada en el año $t$, en ese ámbito" (INE, 2017). 


\subsection{Análisis de la estacionariedad de las series.}

Para cada serie que interviene en el modelo se va a analizar si se cumplen las condiciones para que sea una serie estacionaria. Si la serie es estacionaria en media, es decir, que la serie no tiene tendencia, y por tanto su media no depende de $t E\left(Y_{t}=\theta\right)$ y estacionaria en varianza, cuando mantiene un grado de dispersión similar a lo largo del tiempo, su varianza no depende de $t . \operatorname{Var}\left(Y_{t}\right)=\gamma_{0}$, la serie es estacionaria.

También se analizará si estas series son ergódicas, esto es, si sus covarianzas, $\gamma_{s}=$ $\operatorname{cov}\left(Y_{t}, Y_{t+s}\right)$, decrecen rápidamente hacia cero al aumentar el retardo $s \lim _{s \rightarrow \infty} \gamma_{s}=0$.

Primero se analizarán, ciertos gráficos lineales de cada serie, tras plantear su regresión frente a $t$, variable de tendencia o determinista, así como los correlogramas simples y parciales.

Cuando se realiza la regresión de la variable población ocupada femenina (ocupadas) frente a $t$, se observa que la variable es individualmente significativa ( $p$-valor $=0$ ) y que su coeficiente tiene signo positivo y elevado $(206,77)$. El coeficiente de determinación, $R^{2}$, indica que el $89,95 \%$ de la variabilidad de la variable ocupadas viene explicada por la tendencia determinista.

Figura 1: Regresión de la variable ocupadas frente a $t$.

Dependent Variable: Población ocupada femenina

Sample: 19872015

Includedobservations: 29

\begin{tabular}{lrlrr}
\hline \hline \multicolumn{1}{c}{ Variable } & Coefficient & Std. Error & t-Statistic & Prob. \\
\hline \hline \multicolumn{1}{c}{$\mathrm{t}$} & 206.7713 & 13.30012 & 15.54658 & 0.0000 \\
\multicolumn{1}{c}{$\mathrm{C}$} & 0.899515 & Mean dependentvar & 6108.264 \\
\hline \hline R-squared & 0.895793 & S.D. dependentvar & 1856.329 \\
Adjusted R-squared & 599.2437 & Akaikeinfocriterion & 15.69569 \\
S.E. of regression & 9695511. & Schwarzcriterion & 15.78998 \\
Sum squaredresid & -225.5874 & Hannan-Quinncriter. & 15.72522 \\
Log likelihood & 241.6960 & Durbin-Watson stat & 0.137857 \\
F-statistic & 0.000000 & & \\
Prob(F-statistic) & & & \\
\hline \hline
\end{tabular}

Fuente: Elaboración propia.

En el siguiente gráfico, que proporciona el programa Eviews, se puede observar una clara tendencia determinista creciente, por lo que la media no es constante, sino que crece a lo largo del tiempo, como lo indica la línea verde. En relación con la varianza, se aprecia que 
las fluctuaciones con respecto a la media no son constantes, por lo que la serie también presenta tendencia estocástica.

Gráfico 1: Valores reales y ajustados de las mujeres ocupadas y residuos de la regresión.

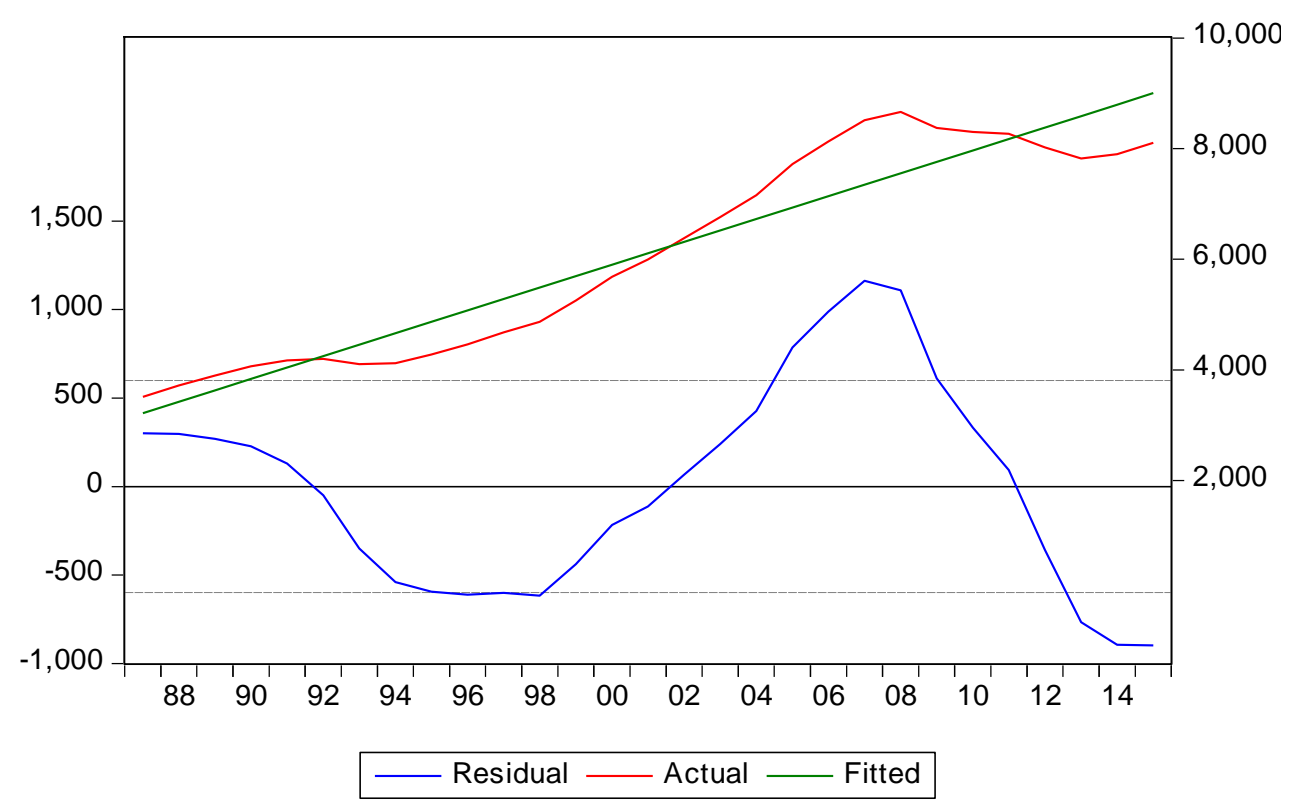

Fuente: Elaboración propia.

En cuanto a los correlogramas, se concluye que la serie no es ergódica, ya que a pesar de que el correlograma parcial decrece rápidamente hacia cero, los coeficientes del correlograma simple son significativamente distintos de cero hasta el séptimo retardo (de un total de 12 representados de forma estadísticamente fiable). 
Figura 2: Correlograma de la serie ocupadas.

\begin{tabular}{|c|c|c|c|c|c|c|c|c|}
\hline Autocorr & relation & Partial Co & relation & & $\mathrm{AC}$ & PAC & Q-Stat & Prob \\
\hline 1 & & 1 & & 1 & 0.934 & 0.934 & 28.002 & 0.000 \\
\hline 1 & & 14 & 1 & 2 & 0.860 & -0.095 & 52.628 & 0.000 \\
\hline 1 & $\square$ & & 1 & 3 & 0.779 & -0.095 & 73.592 & 0.000 \\
\hline 1 & $\square$ & 1 . & 1 & 4 & 0.685 & -0.135 & 90.484 & 0.000 \\
\hline 1 & $\square$ & 1 & 1 & 5 & 0.580 & -0.141 & 103.09 & 0.000 \\
\hline 1 & $\square$ & 1 . & 1 & 6 & 0.468 & -0.114 & 111.65 & 0.000 \\
\hline 1 & $\square$ & 1 단 & 1 & 7 & 0.346 & -0.144 & 116.55 & 0.000 \\
\hline 1 & $\square$ । & 1 든 & 1 & 8 & 0.214 & -0.169 & 118.50 & 0.000 \\
\hline 1 & 1 & 1 С & 1 & 9 & 0.084 & -0.083 & 118.82 & 0.000 \\
\hline 1 & 1 & 1 & I & 10 & -0.037 & -0.034 & 118.88 & 0.000 \\
\hline 1 다 & 1 & 1 & 1 & 11 & -0.146 & -0.015 & 119.94 & 0.000 \\
\hline $1 \square$ & 1 & 1 & 1 & 12 & -0.241 & 0.006 & 123.01 & 0.000 \\
\hline
\end{tabular}

Fuente: Elaboración propia.

A continuación, se realizará el contraste de raíz unitaria para cada una de las series de datos. Para ello, se realiza el Test Aumentado de Dickey-Fuller (ADF), que contrasta la hipótesis nula de que la serie tiene una raíz unitaria, o es integrada de orden uno, frente a la hipótesis alternativa de que es estacionaria o integrada de orden cero. El contraste se hace sobre el siguiente modelo, que también puede incluir tendencia determinista.

$$
\Delta Y_{t}=c+\delta Y_{t-1}+\sum_{i=1}^{\rho} \alpha_{i} \Delta Y_{t-i}+u_{t}
$$

En este caso se incluye la variable de tendencia determinista en la regresión auxiliar del contraste, ya que la serie ocupadas tiene tendencia determinista. Además, hay que tener en cuenta, que al realizar este contraste no se debe detectar autocorrelación, ya que si la hubiera la interpretación no es válida.

Al realizar el test ADF para la variable ocupadas se observa que no existe autocorrelación ya que el estadístico d es 2,25 , próximo a 2 . Además, el último retardo introducido es significativo. El contraste de Dickey-Fuller es válido, concluyéndose que para un p-valor de 0,154 no se rechaza la hipótesis nula, por lo que la serie es no estacionaria, sino integrada de orden uno. 
Figura 3: Test Simple de Dickey-Fuller para la variable ocupadas.

Null Hypothesis: Población ocupada femenina has a unit root

Exogenous: Constant, Linear Trend

Lag Length: 1 (Fixed)

\begin{tabular}{lrrr}
\hline \hline & t-Statistic & Prob. $^{*}$ \\
\hline \hline Augmented Dickey-Fuller test statistic & -2.985109 & $\mathbf{0 . 1 5 4 3}$ \\
\hline Test critical values: & $1 \%$ level & -4.339330 & \\
& $5 \%$ level & -3.587527 & \\
& $10 \%$ level & -3.229230 & \\
\hline \hline
\end{tabular}

*MacKinnon (1996) one-sided p-values.

Augmented Dickey-Fuller Test Equation

Dependent Variable: D(OCUPADAS)

Sample (adjusted): 19892015

Included observations: 27 after adjustments

\begin{tabular}{lrlrl}
\hline \hline \multicolumn{1}{c}{ Variable } & Coefficient & Std. Error & t-Statistic & Prob. \\
\hline \hline OCUPADAS(-1) & -0.141181 & 0.047295 & -2.985109 & 0.0066 \\
D(OCUPADAS(-1)) & 0.887454 & 0.125074 & 7.095449 & 0.0000 \\
$\quad$ C & 423.3836 & 139.4331 & 3.036464 & 0.0059 \\
@TREND("1987") & 30.69275 & 11.01006 & 2.787701 & 0.0105 \\
\hline \hline R-squared & 0.697313 & Mean dependent var & 162.4889 \\
Adjusted R-squared & 0.657832 & S.D. dependent var & 222.1954 \\
S.E. of regression & 129.9735 & Akaike info criterion & 12.70849 \\
Sum squared resid & 388541.7 & Schwarz criterion & 12.90047 \\
Log likelihood & -167.5646 & Hannan-Quinn criter. & 12.76558 \\
F-statistic & 17.66202 & Durbin-Watson stat & $\mathbf{2 . 2 5 1 5 0 9}$ \\
Prob(F-statistic) & 0.000004 & & \\
\hline \hline
\end{tabular}

Fuente: Elaboración propia.

Para las series restantes, se ha realizado el mismo análisis utilizado anteriormente. Primero se estudia un análisis gráfico y después se realiza el test ADF.

Los resultados obtenidos en el análisis se encuentran en el anexo y se concluye que las series, mujeres que trabajan a tiempo parcial (mparcial) e Indicador Coyuntural de Fecundidad (ICF), son series no estacionarias. Sin embargo, la serie tasa bruta de matrícula en nivel superior (educación) es una serie estacionaria. 


\subsection{Análisis de cointegración de las series.}

Como se ha visto en el apartado anterior, todas las series excepto la serie educación, poseen una raíz unitaria. Por este motivo se debe comprobar si las series cointegran ya que todas las series no son estacionarias. La serie educación entra en el modelo sin crear ningún problema. Como las series restantes, es decir, ocupadas, mparcial e ICF, son integradas de orden uno, es decir, que tienen el mismo orden de integración, las variables pueden cointegrar. Si esto sucede, existe una relación de equilibrio a largo plazo entre ellas.

Para realizar este análisis, se empieza estimando la ecuación que sigue por mínimos cuadrados ordinarios (MCO). En esta ecuación se ha incluido la variable tendencia ya que existe en todas las series excepto en la serie ICF. Además, se ha incluido una variable ficticia (D1), que recoge un cambio estructural. Es una variable dicotómica que toma el valor 0 desde 1987 hasta el año 2003 y 1 desde el año 2004 hasta 2015. Se introduce en un primer momento de forma aditiva y multiplicativa solo para la variable mparcial, ya que el cambio de pendiente era más significativo para esa variable. Sin embargo, tras realizar el análisis de cointegración se obtenía una regresión espuria y por ello se concluye incluir la variable ficticia en el modelo de forma aditiva y multiplicativa para las variables mujeres que trabajan a tiempo parcial y para las mujeres matriculadas en educación superior.

Finalmente, la ecuación propuesta es:

$$
\begin{aligned}
\text { ocupadas }_{t} & =\alpha+\beta_{1} \text { mparcial }_{t}+\beta_{2} \text { educacion }_{t}+\beta_{3} i c f_{t}+\beta_{4} t+D 1 \\
& + \text { mparcial }_{t} * D 1+\text { educacion }_{t} * D 1+\varepsilon_{t}
\end{aligned}
$$

Con esta ecuación se han obtenido los siguientes resultados:

Figura 4: Regresión mediante MCO de la variable dependiente frente a las explicativas.

Dependent Variable: OCUPADAS

Sample: 19872015

Includedobservations: 29

\begin{tabular}{crrrr}
\hline \hline Variable & Coefficient & Std. Error & t-Statistic & Prob. \\
\hline \hline C & -7621.807 & 1215.710 & -6.269427 & 0.0000 \\
$\begin{array}{c}\text { Mujeres a tiempo parcial } \\
\text { \% matrícula femenina en }\end{array}$ & -3.947263 & 1.110689 & -3.553888 & 0.0019 \\
nivel superior & 174.2246 & 36.55481 & 4.766121 & 0.0001 \\
Fecundidad & 5179.741 & 474.6056 & 10.91378 & 0.0000 \\
t & -4.060034 & 59.76743 & -0.067931 & 0.9465 \\
D1 & 6547.512 & 461.6461 & 14.18297 & 0.0000 \\
$\begin{array}{c}\text { Mujeres a tiempo } \\
\text { parcial*D1 }\end{array}$ & 5.234998 & 1.150026 & 4.552068 & 0.0002 \\
\% matrícula femenina en & -175.3753 & 22.56275 & -7.772782 & 0.0000 \\
nivel superior *D1 & & & &
\end{tabular}




\begin{tabular}{lrlr}
\hline \hline R-squared & 0.996960 & Mean dependentvar & 6108.264 \\
Adjusted R-squared & 0.995947 & S.D. dependentvar & 1856.329 \\
S.E. of regression & 118.1768 & Akaikeinfocriterion & 12.61119 \\
Sum squaredresid & 293280.7 & Schwarzcriterion & 12.98838 \\
Log likelihood & -174.8623 & Hannan-Quinncriter. & 12.72932 \\
F-statistic & 983.9744 & Durbin-Watson stat & 1.750419 \\
Prob(F-statistic) & 0.000000 & & \\
\hline \hline
\end{tabular}

Fuente: Elaboración propia.

Se trata de una regresión muy buena, ya que los regresores conjuntamente explican casi el $100 \%$ de los datos de la variabilidad de la variable dependiente. Además, al realizar el contraste de la $t$ de Student, se observa que todas las variables explicativas son significativas, con p-valores menores a 0,05 , lo que permite rechazar la hipótesis nula. Sin embargo, la tendencia es claramente no significativa, pero es necesaria para evitar que la regresión sea espuria.

Siguiendo con el análisis de cointegración, en la segunda etapa se trata de analizar la estacionariedad de los residuos. Para ello se generan los residuos correspondientes a la Ecuación 1 ya que se ha concluido que es la más apropiada. Si tras el estudio estos resultan ser estacionarios, las variables cointegrarían y existiría una relación de equilibrio de largo plazo entre ellas. En cambio, si los residuos son no estacionarios, las variables no cointegrarían y estaríamos ante una regresión espuria, en la que se puede estudiar solamente el equilibrio a corto plazo.

Los residuos tienen media cero por definición, por lo que no tienen tendencia determinista. En cuanto a los correlogramas de los residuos, se puede observar cómo la serie sí es ergódica ya que, para ambos correlogramas, el simple y el parcial, los coeficientes de autocorrelación se encuentran dentro de las bandas: todos los coeficientes teóricos son nulos. 
Figura 5: Correlograma de los residuos.

Sample: 19872015

Included observations: 29

\begin{tabular}{|c|c|c|c|c|c|c|c|c|}
\hline \multicolumn{2}{|c|}{ Autocorrelation } & \multicolumn{3}{|c|}{ Partial Correlation } & \multirow{2}{*}{$\frac{A C}{0.107}$} & \multirow{2}{*}{$\frac{\text { PAC }}{0.107}$} & \multirow{2}{*}{$\frac{\text { Q-Stat }}{0.3657}$} & \multirow{2}{*}{$\begin{array}{c}\text { Prob } \\
0.545\end{array}$} \\
\hline 1 & 1 & & । & 1 & & & & \\
\hline 1 & I & & 1 & 2 & -0.040 & -0.052 & 0.4195 & 0.811 \\
\hline 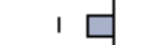 & 1 & 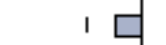 & I & 3 & -0.184 & -0.176 & 1.5896 & 0.662 \\
\hline 1 & 1 & 14 & 1 & 4 & -0.131 & -0.099 & 2.2114 & 0.697 \\
\hline 15 & 1 & 1 & 1 & 5 & -0.064 & -0.059 & 2.3655 & 0.797 \\
\hline 1 & 1 & 1 다 & 1 & 6 & -0.091 & -0.127 & 2.6864 & 0.847 \\
\hline 1 다 & 1 & 1 마 & 1 & 7 & -0.133 & -0.175 & 3.4136 & 0.844 \\
\hline 1 - & 1 & 14 & 1 & 8 & -0.137 & -0.183 & 4.2205 & 0.837 \\
\hline 17 & 1 & 1 & । & 9 & -0.007 & -0.080 & 4.2228 & 0.896 \\
\hline 1 & 1 & 1 나 & 1 & 10 & 0.021 & -0.109 & 4.2439 & 0.936 \\
\hline 1 & 1 & $1 \square$ & । & 11 & -0.004 & -0.160 & 4.2446 & 0.962 \\
\hline 1 & 1 & 1 디 & I & 12 & 0.024 & -0.110 & 4.2739 & 0.978 \\
\hline
\end{tabular}

Fuente: Elaboración propia.

Por último, se aplica el test de Dickey-Fuller a los residuos. En esta ocasión, se obtiene un valor de 1,98 para el estadístico de d de Durbin-Watson, por lo que no existe autocorrelacíon y el test se puede interpretar. Además, no ha sido necesario introducir ningún retardo de las primeras diferencias. Sin embargo, no es válido utilizar el p-valor propuesto en Eviews, dado que corresponde a unos valores críticos que no son correctos para este tipo de análisis. Por ello, se utilizarán los propuestos en las tablas de Davidson y Makinnon. En la ecuación de regresión, de posible cointegración, se introdujo la constante, la tendencia y tres variables integradas de orden uno. Por tanto, para un nivel de significación del $5 \%$, el valor crítico de referencia es de -4,12. Como el estadístico de Dickey-Fuller es de -4,549, que es menor al valor crítico, se rechaza la hipótesis nula, bajo la cual los residuos son no estacionarios. De modo que los residuos son estacionarios y se concluye que las variables cointegran y que la regresión planteada no es espuria, sino que existe una relación de equilibrio a largo plazo entre las variables planteadas.

Figura 6: Test Simple de Dickey-Fuller para los residuos MCO

Null Hypothesis: RESID01 has a unit root

\begin{tabular}{lrrr}
\hline \hline & t-Statistic & Prob. $^{*}$ \\
\hline \hline \multicolumn{2}{l}{ Augmented Dickey-Fuller test statistic } & -4.549863 & 0.0012 \\
\hline Test criticalvalues: & $1 \%$ level & -3.689194 & \\
& $5 \%$ level & -2.971853 & \\
$10 \%$ level & -2.625121 & \\
\hline \hline
\end{tabular}

*MacKinnon (1996) one-sided p-values.

Augmented Dickey-Fuller Test Equation 
Dependent Variable: D(RESID01)

Sample (adjusted): 19882015

Includedobservations: 28 afteradjustments

\begin{tabular}{lrlrr}
\hline \hline \multicolumn{1}{c}{ Variable } & Coefficient & Std. Error & t-Statistic & Prob. \\
\hline \hline \multicolumn{1}{c}{ RESID01(-1) } & -0.891357 & 0.195909 & -4.549863 & 0.0001 \\
\multicolumn{1}{c}{ C } & 1.931374 & 19.82184 & 0.097437 & 0.9231 \\
\hline \hline R-squared & 0.443270 & Mean dependentvar & -0.662498 \\
Adjusted R-squared & 0.421857 & S.D. dependentvar & 137.8877 \\
S.E. of regression & 104.8439 & Akaikeinfocriterion & 12.21157 \\
Sum squaredresid & 285798.5 & Schwarzcriterion & 12.30673 \\
Log likelihood & -168.9620 & Hannan-Quinncriter. & 12.24066 \\
F-statistic & 20.70125 & Durbin-Watson stat & $\mathbf{1 . 9 8 9 6 6 5}$ \\
Prob(F-statistic) & 0.000110 & & \\
\hline \hline
\end{tabular}

Fuente: Elaboración propia.

\subsection{Modelo Propuesto}

Tras los resultados obtenidos en los apartados anteriores, donde finalmente las variables cointegraban se plantea el modelo que recoge el equilibrio a largo plazo.

Como se ha visto, este modelo se corresponde con el modelo presentado en la Ecuación 1. Se trata de un modelo no espurio, en el que si es posible aplicar la teoría econométrica asintótica.

$$
\begin{aligned}
\text { ocupadas }_{t}=\alpha & +\beta_{1} \text { mparcial }_{t}+\beta_{2} \text { educacion }_{t}+\beta_{3} \text { icf }_{t}+\beta_{4} t+D 1+\text { mparcial }_{t} * D 1 \\
& + \text { educacion }_{t} * D 1+\varepsilon_{t}
\end{aligned}
$$

Como ya se ha dicho, esta ecuación presenta un buen ajuste, ya que explica casi el $100 \%$ de la varianza de la variable dependiente.Se comenzará analizando si en este modelo existe o no autocorrelación. El correlograma mostrado en la figura 5 muestra que los coeficientes de correlación simple y parcial no salen de las bandas, por lo que parece que en el modelo no existe autocorrelación. Para confirmar este hecho, se realiza el contraste de Breush-Godfrey, que contrasta la hipótesis nula de ausencia de autocorrelación frente la existencia de autocorrelación. Hacemos sólo el contraste con un retardo, ya que hemos visto que en el correlograma los coeficientes no salen de las bandas, en ningún retardo.Para un pvalor de 0,5247 claramente no se rechaza la hipótesis nula y se concluye que no existe autocorrelación en el modelo

Figura 6: Test de Breusch-Godfrey con un retardo.

Breusch-Godfrey Serial Correlation LM Test:

\begin{tabular}{llll}
\hline \hline F-statistic & 0.282959 & Prob. $F(1,20)$ & 0.6006 \\
Obs*R-squared & 0.404567 & Prob. Chi-Square(1) & $\mathbf{0 . 5 2 4 7}$ \\
\hline \hline
\end{tabular}

Fuente: Elaboración propia. 
Como se ha visto en el apartado anterior, todas las variables explicativas del modelo son significativas, excepto la tendencia. Sin embrago, el estadístico $\mathrm{F}$ del contraste de significación conjunta muestra que son conjuntamente significativas, con un $\mathrm{p}$-valor igual a 0.

Los signos que acompañan a los coeficientes del modelo vienen indicados en la siguiente tabla.

Tabla 1: Signos de los coeficientes del modelo antes y después del cambio estructural.

\begin{tabular}{ccccc}
\hline & Constante & mparcial & educación & ICF \\
\hline Hasta & Negativo & Negativo & Positivo & Positivo \\
$\mathbf{2 0 0 3}$ & $-7621,80$ & $-3,94$ & 174,22 & 5179,74 \\
\hline Desde & Negativo & Positivo & Negativo & Positivo \\
$\mathbf{2 0 0 4}$ & $-7621,8+6547,51$ & $-3,94+5,23$ & $174,22-175,37$ & 5179,74 \\
\hline
\end{tabular}

Fuente: Elaboración propia.

Introducir la variable ficticia de forma aditiva en el modelo hace que cambie el término independiente. En este caso, para ambos periodos, el signo del coeficiente es negativo. Sin embargo, desde el año 2004, el valor esperado de mujeres ocupadas aumenta por el hecho de presentar el cambio estructural en ese año. El término constante negativo carece de interpretación económica, pero es necesario para conseguir el mejor ajuste global del modelo.

También se introduce la variable ficticia de forma multiplicativa, para las variables mparcial y educación, debido al cambio estructural. El signo del coeficiente que acompaña a la variable mujeres que trabajan a tiempo parcial es negativo para el primer periodo, por tanto, el trabajo a tiempo parcial tiene un efecto negativo sobre las mujeres que tienen un trabajo. Esta circunstancia se explica por el "efecto desánimo" ya que, al no encontrar puestos de trabajo a jornada completa, deciden dejar de buscar y no trabajar. Sin embargo, en el segundo periodo, el coeficiente cambia de signo y se vuelve positivo porque muchas mujeres aceptan seguir trabajando a tiempo parcial o incorporarse con este tipo de jornada ante la alternativa de no tener trabajo. 
Por otro lado, el signo del coeficiente de la variable educación hasta el año 2003 es positivo, lo que concuerda con la evidencia empírica. Cuando aumenta en una unidad el número de mujeres matriculadas en nivel superior, el número de mujeres que trabajan crece. No obstante, a partir del año 2004, el signo cambia y se vuelve negativo, hecho que no concuerda con el resultado de otras investigaciones. Sin embargo, cabe pensar que un aumento del número de mujeres matriculadas en educación superior disminuye el número de mujeres que están trabajando debido a que, en los últimos años, muchas mujeres han aumentado el número de años que dedican a estudiar y así, obtener una mayor cualificación para poder hacer frente a la demanda de mercado de trabajo. Por ello, en los últimos años, la incorporación de la mujer, especialmente de las mujeres jóvenes, a un puesto de trabajo, se retrasa en aras de una mayor formación académica.

Respecto a la variable número medio de hijos por mujer (ICF), el coeficiente es el mismo y positivo, para ambos periodos, ya que a esta variable no le afecta el cambio estructural, es decir que, al aumentar el número medio de hijos que tendría una mujer, el número de mujeres trabajando sube, hecho que no parece concordar con los resultados de otros estudios analizados. Sin embargo, esto se explica porque en la actualidad son las propias madres las que quieren seguir trabajando una vez que han tenido un hijo y buscan otras alternativas para no tener que abandonar su puesto de trabajo. Además, tras la reforma laboral del año 2006, que incentivaba a las empresas a través de bonificaciones por los contratos indefinidos de mujeres que tuvieran un contrato suspendido por maternidad o por excedencia de cuidado de los hijos, las mujeres se han visto menos forzadas a abandonar el mercado laboral.

El signo del coeficiente de la tendencia no se estudiará, al ser la tendencia no significativa individualmente.

\section{Discusión y conclusiones}

A pesar de que hoy en día la población femenina es superior a la masculina, la tasa de ocupación de las mujeres españolas sigue siendo inferior a la de los hombres y se sitúa a la cola de los países de la Unión Europea.

A lo largo de las últimas décadas, la participación femenina en el mercado laboral ha aumentado debido fundamentalmente al mayor número de años que las mujeres dedican a los estudios, por lo que obtienen una mayor cualificación; al aumento de la edad de maternidad, y a una disminución en el número medio de hijos. Este cambio ha generado un gran impacto social, aunque en la actualidad siguen siendo las mujeres las que realizan la mayoría de las tareas no remuneradas; por este motivo, en algunos casos, buscan trabajos 
que les permitan conciliar su vida laboral con la familiar y ocupan muchos de los puestos de trabajo a tiempo parcial. Sin embargo, la mayoría de ellas trabajan en este tipo de empleo por no poder encontrar uno a jornada completa, situación que no se da en países del norte de Europa.

Es importante señalar que, a pesar de los avances conseguidos en materia de trabajo fundamentalmente en el ámbito legislativo, tras la reforma laboral de 2012 la situación de las mujeres en el mercado de trabajo ha mejorado, pero sigue existiendo una destacada diferencia salarial entre hombres y mujeres que no se ha conseguido reducir.

Todo esto provoca una menor independencia económica y una mayor inestabilidad del empleo femenino, con menores oportunidades de ascenso a lo largo de su vida laboral. Por ello, es necesario mejorar el acceso de mujeres a altos puestos, reformulando las políticas activas de empleo y las políticas de incentivos al empleo femenino, ya que no son efectivas. De igual manera, la negociación y los convenios colectivos deberían mejorar la inserción laboral de las mujeres, al igual que las políticas públicas de lucha contra la discriminación (Informe "Participación Laboral de las Mujeres en España", 2016). Además, se debe en cuenta que los trabajos donde existe igualdad entre hombres y mujeres presentan una mayor eficiencia y productividad (Salinas y Romaní, 2016).

En cuanto al modelo planteado, se comprobó que tres de las series elegidas eran integradas de orden uno y, por tanto, no estacionarias. Por ello, se analizó si la relación de equilibrio a largo plazo entre las variables era espuria o si, por el contrario, cointegraban. Finalmente, se dedujo que las variables cointegraban, lo que permitió plantear un modelo que recogía la relación de largo plazo entre ellas. Este modelo contaba con un buen ajuste, no existía autocorrelación y las variables eran conjuntamente significativas, excepto la variable de tendencia, que a pesar de no ser individualmente significativa, fue necesaria para obtener una regresión no espuria.

Al analizar el signo de los coeficientes del modelo, se advirtió que las variables explicativas influían de forma distinta, antes y después del cambio estructural. Se observó que la variable mparcial influía de forma negativa sobre las mujeres ocupadas, resultado que se explica por el "efecto desánimo", y que después de dicho cambio tenía un efecto positivo, es decir que, desde el año 2004, las mujeres prefieren trabajar, aunque sea a tiempo parcial. Algo parecido ocurría con la variable educación: antes del cambio, influía positivamente sobre la variable dependiente y después del cambio, de forma negativa, hecho que no concuerda con los resultados de otros estudios, pero explicado porque las mujeres han aumentado sus años de estudio para obtener una mayor cualificación y por ello, el número de mujeres ocupadas disminuye. 
La variable número medio de hijos por mujer no se vio afectada por el cambio estructural, pero al tener su coeficiente un signo positivo, parecía que no concordaba con la evidencia empírica. No obstante, este resultado se puede explicar por las ayudas recibidas tras la Reforma del año 2006 y por el hecho de que las mujeres prefieren seguir trabajando. Este trabajo pretende constituir una pequeña aportación al estudio de un tema esencial para el avance social.

\section{Referencias bibliográficas}

Alonso Antón, A., Fernández Sainz, A., \& Rincón Diez, V. (2015). Análisis de la actividad femenina y la fecundidad en España mediante modelos de elección discreta. Lecturas de Economía, (82), pp. 127-157.

Cebrián López, I., \& Moreno Raymundo, G. (2008). La situación de las mujeres en el mercado de trabajo español. Desajustes y retos. Economía Industrial, (367), pp. 121-137.

Cebrián López, I., \& Moreno Raymundo, G. (2013). Mujeres, hogares y mercado de trabajo en la obra de Luis Toharia. Revista de Economía Laboral, (10), pp. 104-123.

Comisión Europea-Dirección de Justicia. (2014). Cómo combatir la brecha salarial entre hombres y mujeres en la Unión Europea. Recuperado de: http://ec.europa.eu/justice/genderequality/files/gender_pay_gap/140319_gpg_es.pdf [última consulta: 31/03/2017]

De Pablos Escobar, L. (sin fecha). Las mujeres y el mercado de trabajo. La participación laboral femenina de: http://pmayobre.webs.uvigo.es/06/arch/profesorado/dolores_ferrero/mujer_trabaj o.pdf [última consulta: 01/05/2017].

Elneser Montesinos, D. (2015). Desigualdades de la mujer en su incorporación al mercado laboral español. Universidad de La Laguna, San Cristóbal de La Laguna. Recuperado de: http://riull.ull.es/xmlui/handle/915/1340[última consulta: 04/04/2017].

Ferrada Bórquez, L.M., \& Zarzosa Espina, P. (2010). Diferencias Regionales en la Participación Laboral Femenina en Chile. Cuadernos de economía, 47 (136), pp. 249-272. Recuperado de: https://dx.doi.org/10.4067/S071768212010000200005[última consulta: 15/05/2017].

García Echegaray, J. (28 de octubre de 2016). España presenta una mayor igualdad salarial entre hombres y mujeres que la media de la UE. Libre Mercado. Recuperado de: https://www.libremercado.com/2016-11-28/espana-presenta-una-mayor- 
igualdad-salarial-entre-hombres-y-mujeres-que-la-media-de-la-ue-1276587133/ [última consulta: 07/012/2017].

González Güemes, I. Prieto Alaiz, M. Pérez Dominguez, C. (1999). La participación laboral de la mujer en Castilla y León. Revista de investigación económica y social de Castilla y León, (2), pp. 83-98. Recuperado de: https://dialnet.unirioja.es/servlet/articulo?codigo=202072 [última consulta: 25/04/2017]

Guevara Ramirez, K. (2014). La participación de la mujer en el mercado laboral del área metropolitana de Cali en diciembre del 2012. Una aproximación a los determinantes socioculturales: un caso experimental. Universidad del Valle, Santiago de Cali. Recuperado de: http://bibliotecadigital.univalle.edu.co/handle/10893/7616[última consulta: 13/04/2017]

Hernández Colino G. (2015). Brecha salarial de género en la Unión Europea. Universidad de La Laguna, San Cristóbal de La Laguna. Recuperado de: https://riull.ull.es/xmlui/handle/915/2724[última consulta: 28/03/2017].

Instituto Nacional de Estadística (2017): Actividades de trabajo no remunerado. Introducción, definiciones y comentarios. Disponible en: http://www.ine.es/ss/Satellite?L=es_ES\&c=INESeccion_C\&cid=1259925472542\& $\mathrm{p}=1254735110672$ \&pagename $=$ ProductosYServicios\%2FPYSLayout\&param1 $=P$ YSDetalle\&param3=1259924822888[última consulta: 05/05/2017].

Instituto Nacional de Estadística (2017): Participación de los trabajadores a tiempo parcial y de los trabajadores con contrato temporal. Introducción, definiciones y comentarios. Disponible en: http://www.ine.es/ss/Satellite?L=es_ES\&c=INESeccion_C\&cid=1259925461713\& $\mathrm{p}=1254735110672$ \&pagename=ProductosYServicios\%2FPYSLayout\&param3=1 259924822888[última consulta: 12/05/2017].

Instituto Nacional de Estadística (2017): Salario anual medio, mediano y modal. Salario por hora. Brecha salarial de género (no ajustada) en salarios por hora. Introducción, definiciones y comentarios. Disponible en: http://www.ine.es/ss/Satellite?L=es_ES\&c=INESeccion_C\&cid=1259925408327\& $\mathrm{p}=1254735110672 \&$ pagename=ProductosYServicios\%2FPYSLayout[última consulta: 14/04/2018].

Martínez Granado, M. (2001). Oferta de trabajo femenina en España: un modelo empírico aplicado a mujeres casadas. Cuadernos económicos de ICE, 66, pp.129-152. 
Recuperado de: http://www.revistasice.com/cachepdf/cice_66_129152 _8402e5e30be688293cf25dc9739ed752.pdf [última consulta: 10/05/2017].

Millán Vázquez de la Torre, Ma․ G., Santos Pita, M. P., \& Pérez Naranjo, L. (2015). Análisis del mercado laboral femenino en España: Evolución y factores socioeconómicos determinantes del empleo. Papeles de población, 21(84), pp.197-225. Recuperado de: http://www.scielo.org.mx/scielo.php?script=sci_arttext\&pid=S140574252015000200008 [última consulta: 20/02/2017].

Oelz, M.; Olney, S.; \& Tomei, M. (2013). Igualdad Salarial: Guía Introductoria. Oficina Internacional del Trabajo. Recuperado de: http://www.ilo.org/wcmsp5/groups/public/---ed_norm/--normes/documents/publication/wcms_223157.pdf [última consulta: 10/03/2017].

Ortiz García, P. (2014). El trabajo a tiempo parcial ¿Una alternativa para la mujer en tiempos de crisis? Sociología del trabajo. 82 pp. 73-92.

Real Decreto Legislativo 2/2015, de 23 de octubre, por el que se aprueba el texto refundido de la Ley de Estatuto de los Trabajadores. Boletín Oficial del Estado. Madrid, 24 de octubre de 2015, pp. 100224-100308.

Salinas, P. \& Romaní, G. (2016). Hegemonía masculina, freno en equipos mixtos en la minería chilena. Revista mexicana de sociología, 78 (3), 469-496. Recuperado de: http://www.scielo.org.mx/scielo.php?script=sci_arttext\&pid=S018825032016000300469 [última consulta: 15/09/2017].

Secretaría de Estado de Empleo. Ministerio de Empleo y Seguridad Social. (2016). La situación de las mujeres en el mercado de trabajo en 2016. Recuperado de: http://www.ces.es/documents/10180/3557409/Inf0516.pdf [última consulta: 8/03/2017].

\section{Anexo}

- Mujeres que trabajan a tiempo parcial (mparcial).

Figura 1: Regresión de la variable mujeres que trabajan a tiempo parcial frente a $t$.

Dependent Variable: Mujeres a tiempo parcial

Sample: 19872015

Included observations: 29

\begin{tabular}{ccccc}
\hline \hline \multicolumn{1}{c}{ Variable } & Coefficient & Std. Error & t-Statistic & Prob. \\
\hline \hline $\mathrm{t}$ & 68.60369 & 3.531339 & 19.42710 & 0.0000 \\
$\mathrm{C}$ & 219.9069 & 57.59440 & 3.818199 & 0.0007 \\
\hline \hline R-squared & 0.933236 & Mean dependentvar & 1180.359 \\
Adjusted R-squared & 0.930764 & S.D. dependentvar & 604.6731
\end{tabular}




\begin{tabular}{lrll} 
S.E. of regression & 159.1063 & Akaikeinfocriterion & 13.04349 \\
Sum squaredresid & 683500.2 & Schwarzcriterion & 13.13779 \\
Log likelihood & -187.1307 & Hannan-Quinncriter. & 13.07303 \\
F-statistic & 377.4124 & Durbin-Watson stat & 0.405701 \\
Prob(F-statistic) & 0.000000 & & \\
\hline
\end{tabular}

Fuente: Elaboración propia.

Figura 2: Valores reales y ajustados de las mujeres que trabajan a tiempo parcial y residuos de la regresión.

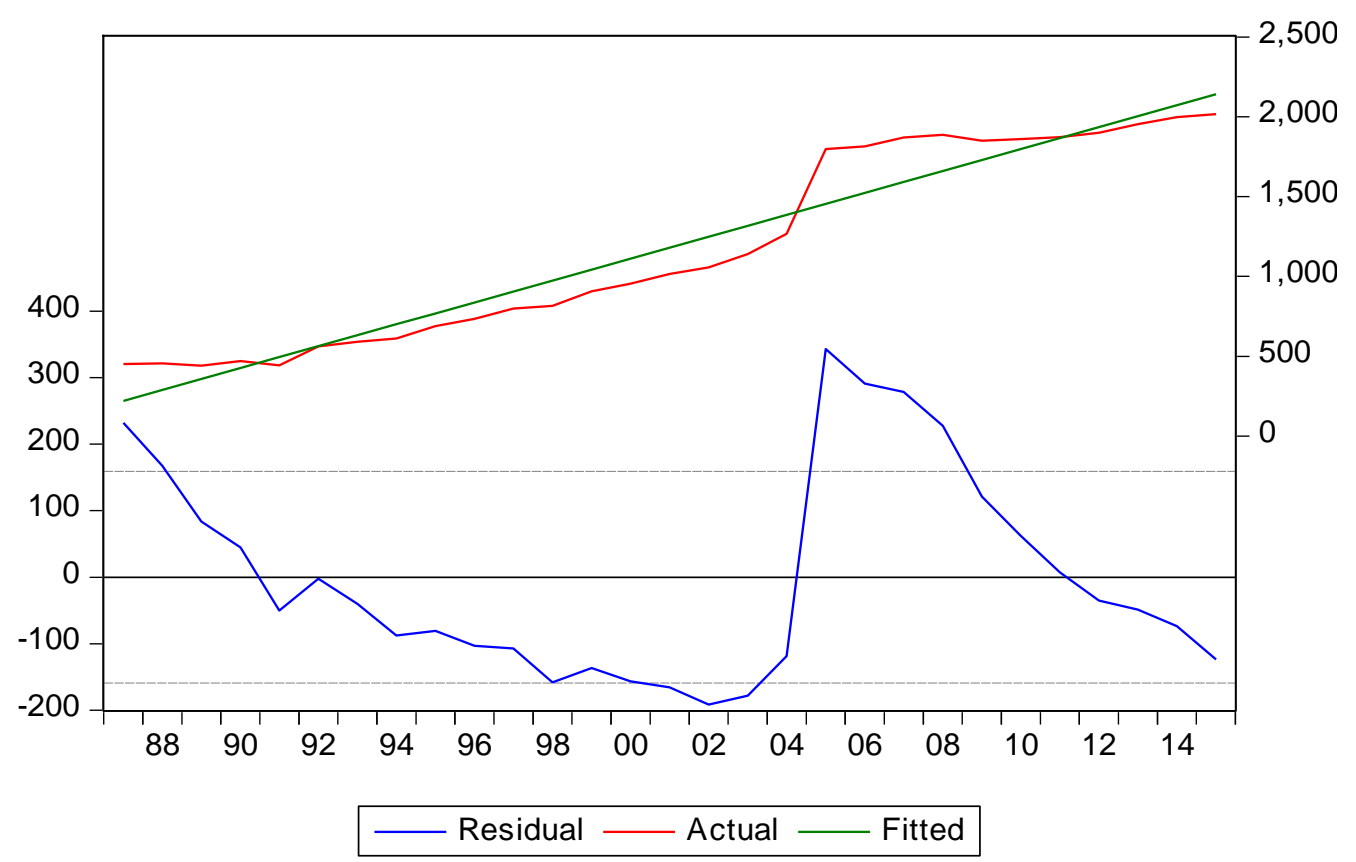

Fuente: Elaboración propia.

Figura 3: Correlograma de la serie mujeres que trabajan a tiempo parcial.

Sample: 19872015

Included observations: 29

\begin{tabular}{|c|c|c|c|c|c|c|c|c|}
\hline \multicolumn{2}{|c|}{ Autocorrelation } & \multicolumn{3}{|c|}{ Partial Correlation } & \multirow{2}{*}{$\frac{A C}{0.922}$} & \multirow{2}{*}{$\frac{\text { PAC }}{0.922}$} & \multirow{2}{*}{$\begin{array}{l}\text { Q-Stat } \\
27.309\end{array}$} & \multirow{2}{*}{$\frac{\text { Prob }}{0.000}$} \\
\hline 1 & & 1 & & 1 & & & & \\
\hline 1 & & 1 다 & 1 & 2 & 0.835 & -0.105 & 50.518 & 0.000 \\
\hline 1 & 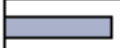 & & 1 & 3 & 0.739 & -0.103 & 69.389 & 0.000 \\
\hline 1 & $\longmapsto$ & 1 & 1 & 4 & 0.642 & -0.055 & 84.198 & 0.000 \\
\hline 1 & $\square$ & ㄴ. & I & 5 & 0.540 & -0.090 & 95.113 & 0.000 \\
\hline 1 & $\square$ & 1 & 1 & 6 & 0.441 & -0.042 & 102.71 & 0.000 \\
\hline 1 & 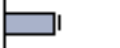 & 14 & 1 & 7 & 0.337 & -0.101 & 107.36 & 0.000 \\
\hline 1 & $\square$ । & 1 - & 1 & 8 & 0.226 & -0.131 & 109.54 & 0.000 \\
\hline 1 & $5 \quad 1$ & 1 Ц & I & 9 & 0.112 & -0.099 & 110.11 & 0.000 \\
\hline 1 & 1 & 14 & 1 & 10 & -0.001 & -0.101 & 110.11 & 0.000 \\
\hline 14 & 1 & 1 - & 1 & 11 & -0.116 & -0.122 & 110.78 & 0.000 \\
\hline $1 \square$ & 1 & 1 & $\sqsupseteq$ 口 & 12 & -0.190 & 0.168 & 112.70 & 0.000 \\
\hline
\end{tabular}

Fuente: Elaboración propia. 
- Tasa bruta de matrícula en nivel superior (educación).

Figura 4: Regresión de la variable mujeres que han alcanzado estudios superiores frente a $t$.

Dependent Variable: \% matrícula femenina en nivel superior

Sample: 19872015

Includedobservations: 29

\begin{tabular}{lrlrr}
\hline \hline \multicolumn{1}{c}{ Variable } & Coefficient & Std. Error & t-Statistic & Prob. \\
\hline \hline \multicolumn{1}{c}{$\mathrm{t}$} & 2.497731 & 0.028200 & 88.57055 & 0.0000 \\
\multicolumn{1}{c}{$\mathrm{c}$} & 29.43529 & 0.459936 & 63.99870 & 0.0000 \\
\hline \hline R-squared & 0.996570 & Mean dependentvar & 64.40352 \\
Adjusted R-squared & 0.996443 & S.D. dependentvar & 21.30398 \\
S.E. of regression & 1.270587 & Akaikeinfocriterion & 3.383307 \\
Sum squaredresid & 43.58855 & Schwarzcriterion & 3.477603 \\
Log likelihood & -47.05795 & Hannan-Quinncriter. & 3.412839 \\
F-statistic & 7844.742 & Durbin-Watson stat & 0.690487 \\
Prob(F-statistic) & 0.000000 & & \\
\hline \hline
\end{tabular}

Fuente: Elaboración propia.

Figura 5: Valores reales y ajustados de las mujeres que han alcanzado estudios superiores y residuos de la regresión.

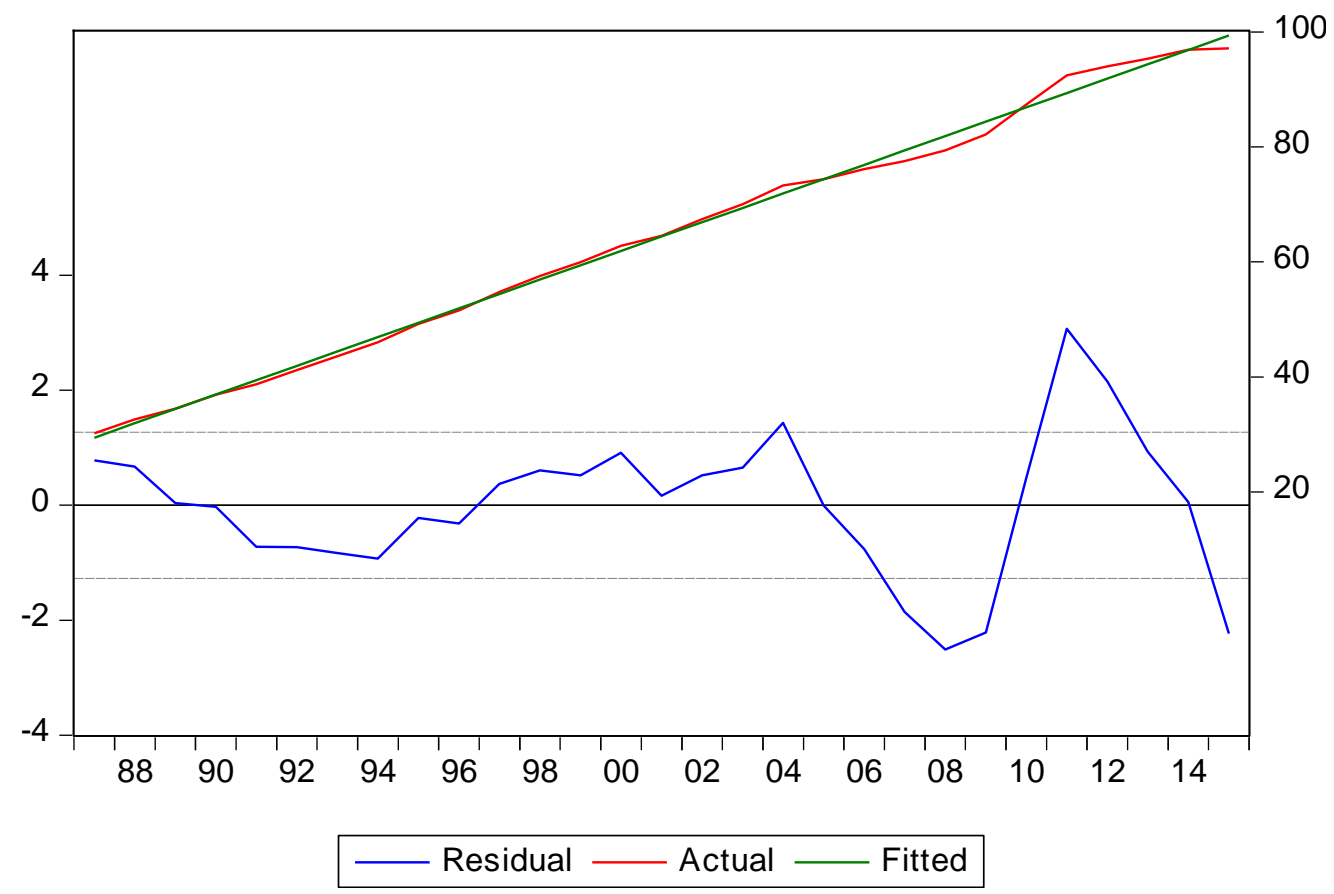

Fuente: Elaboración propia. 
Figura 6: Correlograma de la serie mujeres que han alcanzado estudios superiores.

Sample: 19872015

Included observations: 29

\begin{tabular}{|c|c|c|c|c|c|c|c|c|}
\hline \multicolumn{2}{|c|}{ Autocorrelation } & \multicolumn{3}{|c|}{ Partial Correlation } & \multirow{2}{*}{$\frac{A C}{0.904}$} & \multirow{2}{*}{$\frac{P A C}{0.904}$} & \multirow{2}{*}{$\frac{\text { Q-Stat }}{26.261}$} & \multirow{2}{*}{$\frac{\text { Prob }}{0.000}$} \\
\hline 1 & & 1 & & 1 & & & & \\
\hline 1 & & 1 다 & 1 & 2 & 0.802 & -0.085 & 47.697 & 0.000 \\
\hline 1 & & 15 & 1 & 3 & 0.697 & -0.073 & 64.506 & 0.000 \\
\hline 1 & $\square$ & 10 & 1 & 4 & 0.591 & -0.070 & 77.047 & 0.000 \\
\hline 1 & $\square$ & c & 1 & 5 & 0.482 & -0.077 & 85.749 & 0.000 \\
\hline 1 & $\square$ & 1 & 1 & 6 & 0.383 & -0.018 & 91.485 & 0.000 \\
\hline 1 & ו & 1 & 1 & 7 & 0.294 & -0.019 & 95.020 & 0.000 \\
\hline 1 & $\square$ । & 1 & 1 & 8 & 0.209 & -0.053 & 96.885 & 0.000 \\
\hline 1 & D 1 & 1 & 1 & 9 & 0.128 & -0.052 & 97.617 & 0.000 \\
\hline 1 & 1 & 1 & 1 & 10 & 0.048 & -0.070 & 97.725 & 0.000 \\
\hline 1 & 1 & 1 & 1 & 11 & -0.027 & -0.051 & 97.762 & 0.000 \\
\hline 1 니 & 1 & 1 & 1 & 12 & -0.100 & -0.065 & 98.286 & 0.000 \\
\hline
\end{tabular}

Fuente: Elaboración propia.

- Indicador Coyuntural de Fecundidad (ICF).

Figura 7: Regresión de la variable número medio de hijos por mujer frente a $t$.

Dependent Variable: Fecundidad

Sample: 19872015

Includedobservations: 29

\begin{tabular}{lrlrr}
\hline \hline \multicolumn{1}{c}{ Variable } & Coefficient & Std. Error & t-Statistic & Prob. \\
\hline \hline \multicolumn{1}{c}{$\mathrm{t}$} & 0.000719 & 0.002187 & 0.328861 & 0.7448 \\
\multicolumn{1}{c}{1.289931} & 0.035669 & 36.16435 & 0.0000 \\
\hline \hline R-squared & 0.003990 & Mean dependentvar & 1.300000 \\
Adjusted R-squared & -0.032900 & S.D. dependentvar & 0.096954 \\
S.E. of regression & 0.098536 & Akaikeinfocriterion & -1.730326 \\
Sum squaredresid & 0.262150 & Schwarzcriterion & -1.636030 \\
Log likelihood & 27.08973 & Hannan-Quinncriter. & -1.700794 \\
F-statistic & 0.108149 & Durbin-Watson stat & 0.158858 \\
Prob(F-statistic) & 0.744798 & & \\
\hline \hline
\end{tabular}

Fuente: Elaboración propia. 
Figura 8: Valores reales y ajustados del número medio de hijos por mujer y residuos de la regresión.

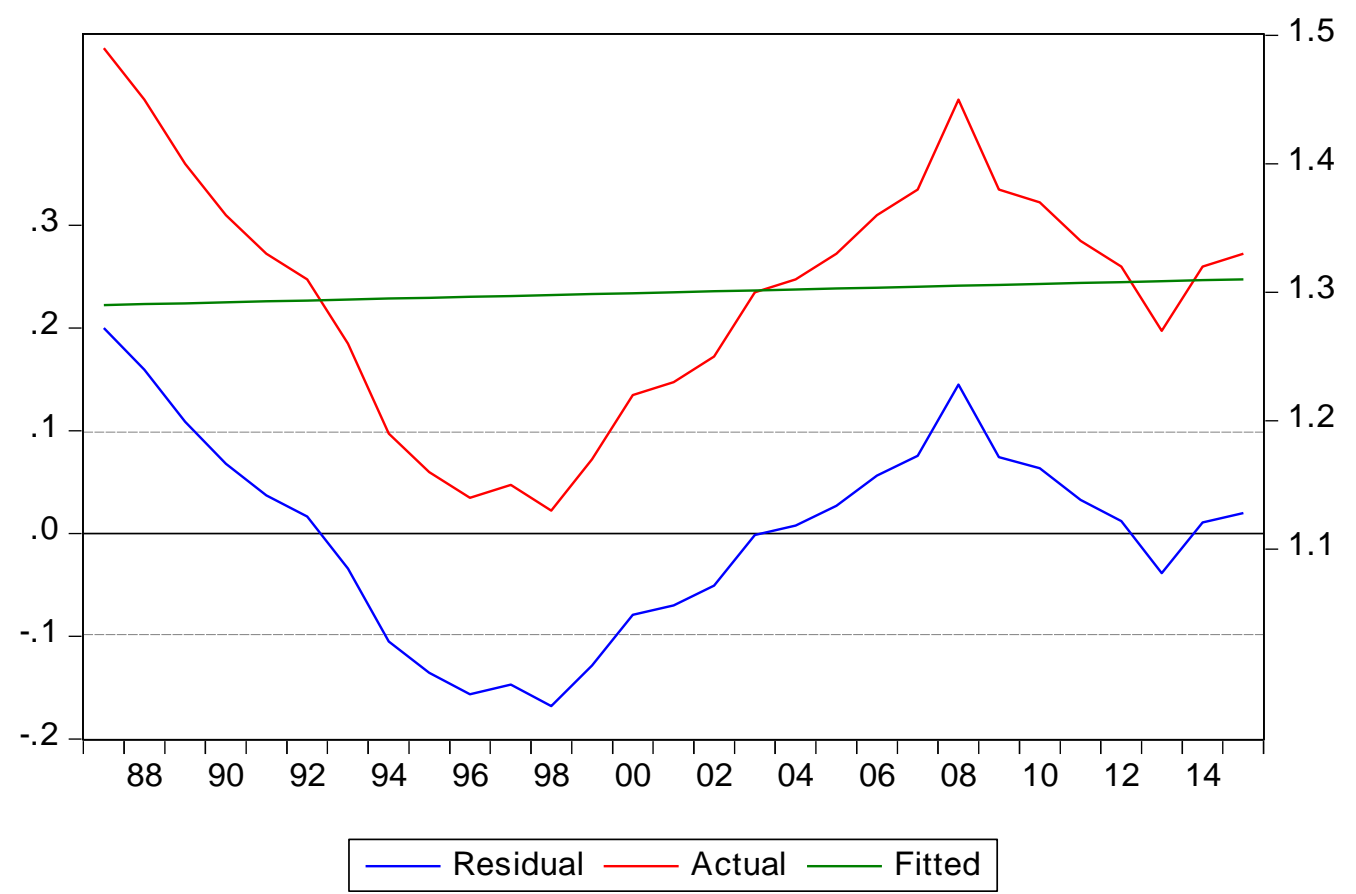

Fuente: Elaboración propia.

Figura 9: Correlograma de la serie número medio de hijos por mujer.

Date: 05/30/17 Time: $20: 11$

Sample: 19872015

Included observations: 29

\begin{tabular}{|c|c|c|c|c|c|c|c|c|}
\hline \multicolumn{2}{|c|}{ Autocorrelation } & \multicolumn{2}{|c|}{ Partial Correlation } & \multicolumn{2}{|r|}{$\mathrm{AC}$} & PAC & Q-Stat & Prob \\
\hline 1 & & 1 & & 1 & 0.843 & 0.843 & 22.842 & 0.000 \\
\hline 1 & 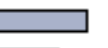 & 1 다 & 1 & 2 & 0.663 & -0.169 & 37.459 & 0.000 \\
\hline 1 & $\square$ & 1 다 & 1 & 3 & 0.465 & -0.163 & 44.932 & 0.000 \\
\hline 1 & $\exists 1$ & 1 口 & 1 & 4 & 0.256 & -0.171 & 47.283 & 0.000 \\
\hline I & 1 & $1 \square$ & 1 & 5 & 0.031 & -0.223 & 47.318 & 0.000 \\
\hline 1 마 & 1 & 1 다 & 1 & 6 & -0.178 & -0.152 & 48.557 & 0.000 \\
\hline & 1 & 14 & 1 & 7 & -0.350 & -0.107 & 53.552 & 0.000 \\
\hline$\square$ & 1 & 1 & 1 & 8 & -0.461 & -0.028 & 62.648 & 0.000 \\
\hline & 1 & 14 & 1 & 9 & -0.533 & -0.095 & 75.424 & 0.000 \\
\hline & 1 & 1 & 1 & 10 & -0.551 & -0.040 & 89.805 & 0.000 \\
\hline & 1 & 1 & 1 & 11 & -0.516 & -0.019 & 103.10 & 0.000 \\
\hline$\square$ & 1 & 1 & 1 & 12 & -0.434 & -0.008 & 113.06 & 0.000 \\
\hline
\end{tabular}

Fuente: Elaboración propia. 